\title{
Prevalence of 25-hydroxyvitamin D deficiency in the acute rehabilitation population following traumatic brain injury
}

\author{
Rosemary Dubiel $^{\mathrm{a}, \mathrm{b}, *}$, Benecia Williams ${ }^{\mathrm{a}, \mathrm{b}}$, Erin Sullivan ${ }^{\mathrm{a}}$, Librada Callender ${ }^{\mathrm{a}, \mathrm{b}}$, \\ Monica Bennett ${ }^{\mathrm{b}}$ and Simon Driver ${ }^{\mathrm{a}, \mathrm{b}}$ \\ ${ }^{a}$ Baylor Scott \& White Institute for Rehabilitation, Dallas, TX, USA \\ ${ }^{\mathrm{b}}$ Baylor University Medical Center at Dallas, Dallas, TX, USA
}

\begin{abstract}
.
OBJECTIVE: To determine the prevalence of vitamin D deficiency among individuals with traumatic brain injury (TBI) undergoing acute inpatient rehabilitation.

SETTING: Inpatient rehabilitation.

PARTICIPANTS: Participants were admitted from November 1st, 2010 through June 30th, 2015 with diagnosis of mildsevere TBI and had serum $25 \mathrm{OH}$ vitamin D levels checked upon admission. 369 out of 845 met inclusion.

DESIGN: Retrospective cohort.

MAIN MEASURES: $25 \mathrm{OH}$ vitamin D, vitamin D treatment.

RESULTS: The majority of patients were male $(67 \%)$, Caucasian $(89 \%)$ with private insurance $(61 \%)$ and a mean age of $43.5 \pm 21.0$ years. $25 \mathrm{OH}$ vitamin D levels were categorized: deficient $(<20 \mathrm{ng} / \mathrm{mL})$, insufficient $(20-29.9 \mathrm{ng} / \mathrm{mL})$, and sufficient ( $\geq 30 \mathrm{ng} / \mathrm{mL}$ ). There were $95(26 \%)$ patients that were deficient, $131(36 \%)$ that were insufficient, and $143(39 \%)$ that were sufficient. Race/ethnicity was found to be significant $(p=0.0145)$ with the largest percentage of Hispanics found in the insufficient and deficient categories, and the largest percentage of Blacks in the deficient category. Most patients with deficient or insufficient vitamin D levels were prescribed vitamin D replacement during their rehab stay $(p<0.0001)$.

CONCLUSION: The majority of patients undergoing acute inpatient rehabilitation were found to have insufficient or deficient vitamin D levels, therefore it should be routinely screened and treated as indicated.
\end{abstract}

Keywords: Brain injuries, vitamin D, rehabilitation

The acute and chronic effects of traumatic brain injury (TBI) represent a significant health concern in the United States (Centers for Disease Control and Prevention, 2014; Faul, Xu, Wald, \& Coronado,

This work was presented as a poster at the American Congress of Rehabilitation Medicine Conference, October 2016, Chicago, IL, USA.

*Address for correspondence: Rosemary Dubiel, DO, Baylor Institute for Rehabilitation at Dallas, 909 North Washington Avenue, Dallas, TX 75246, USA, Tel.: +1 214820 9593; E-mail: rdubiel@bir-rehab.com.
2010), that may lead to deficits in motor function, memory, executive function, mood, sleep, and fatigue (Centers for Disease Control and Prevention, 2014; Jamall et al., 2016). The acute inpatient rehabilitation setting provides the opportunity and environment to begin the rehabilitation process, which includes medical, physical and cognitive treatment. Assessment of nutritional status following injury is also critical during inpatient rehabilitation. Vitamin D is one of the nutrients that may be monitored as it plays a unique role in facilitating cognitive and physical 
functioning in healthy individuals (Gloth \& Tobin, 1995; Houston et al., 2007; Van der Schaft et al., 2013).

Vitamin D is a fat-soluble secosteroid that aids intestinal absorption of calcium, iron, magnesium, phosphate and zinc. Additionally, it plays a vital role in muscle function (Boonen et al., 2006), cardiovascular health (Muscogiuri et al., 2016), cancer prevention (Shan et al., 2016), and reducing risk for diabetes (Grammatiki, Rapti, Karras, Ajjan, \& Kotsa, 2017). Specifically, vitamin D aids protein synthesis in muscles, helps protect against myocardial infarction and cardiovascular disease, regulates cancer stem-like cells, and may improve glycemic control and prevent complications in diabetes (Boonen et al., 2006; Grammatiki et al., 2017; Holick et al., 2011; Muscogiuri et al., 2016; Shan et al., 2016). Within the brain, vitamin D supports neural protection through antioxidant mechanisms, calcium regulation, enhanced nerve conduction, and detoxification mechanisms (Buell \& Dawson-Hughes, 2008). As adequate neural functioning and protection play such a large role in recovery from TBI (Povlishock \& Katz, 2005), these vitamin D mediated protection mechanisms may be especially important in a rehabilitation population.

The rate of vitamin D deficiency, defined as serum $25 \mathrm{OH}$ vitamin D level less than $20 \mathrm{ng} / \mathrm{ml}$, is estimated to be between $25 \%$ and $57 \%$ in the general U.S. population (Holick et al., 2011). Vitamin D deficiency may affect physical and cognitive health, is a risk factor for osteoporosis, osteopenia and bone fractures, and has been associated with musculoskeletal pain and impaired physical function (Boonen et al., 2006; Holick et al., 2011; Muscogiuri et al., 2016). Insufficient (20-29.9 ng/mL) and deficient levels $(<20 \mathrm{ng} / \mathrm{mL})$ of $25 \mathrm{OH}$ vitamin $\mathrm{D}$ have also been associated with poor performance on cognitive tasks, and may also be correlated with dementia diagnosis and related cognitive decline (Van der Schaft et al., 2013).

While numerous studies have investigated vitamin D insufficiency or deficiency in the general population, limited data is available in those undergoing rehabilitation. In a study of rehabilitation patients admitted for brain, spinal cord, and musculoskeletal injuries, Shinchuk and colleagues found that $83 \%$ were vitamin D insufficient or deficient (Shinchuk et al., 2006). Further, in an examination of the functional impact of vitamin D deficiency in rehabilitation populations (e.g., stroke, orthopedic injuries), Kiebzak reported that patients with deficient or insuf- ficient vitamin D levels correlated with significantly lower Functional Independence Measure (FIM) efficiency scores (Kiebzak, Moore, Margolis, Hollis, \& Kevorkian, 2007). These results highlight both increased prevalence of vitamin D deficiency in rehabilitation populations and the functional impact of this deficiency.

While early investigational results highlight the potential issues surrounding insufficient vitamin D in rehabilitation patients, no study has thus far examined the prevalence and functional implications of insufficient or deficient vitamin D among TBI patients undergoing acute inpatient rehabilitation. This is important as inpatient rehabilitation provides an opportunity to treat nutritional imbalances that exist, and vitamin D may have implications for physical and cognitive recovery. Therefore, the present study sought to identify vitamin D prevalence rates in a TBI rehabilitation sample and investigate the association between vitamin D levels and select functional outcomes. A retrospective chart review was performed on patients admitted to a large urban rehabilitation hospital over a four-and-a-half-year span. All patients were diagnosed with a mild to severe TBI.

\section{Methods}

\subsection{Participants}

Seven hundred and ninety-eight medical charts for patients with etiological diagnosis of TBI were obtained from the eRehabData ${ }^{\circledR}$ admissions data portal at a large rehabilitation hospital located within an urban metropolitan area between May 1st, 2011 and June 30th, 2015. Charts were further reviewed for inclusion which were diagnosis of traumatic brain injury and completed vitamin D screening during inpatient rehabilitation via serum 25-hydroxy upon rehabilitation admission. Patients were excluded from the study due to a comorbid or primary diagnosis of spinal cord injury, no etiological diagnosis of TBI, or incomplete chart data. Of the 798 charts initially reviewed, 369 (46.2\%) had vitamin D screening and were eligible for the study (see Fig. 1).

\subsection{Procedures}

IRB approval was obtained prior to beginning the retrospective chart review. Information was extracted from electronic medical records contained in the 


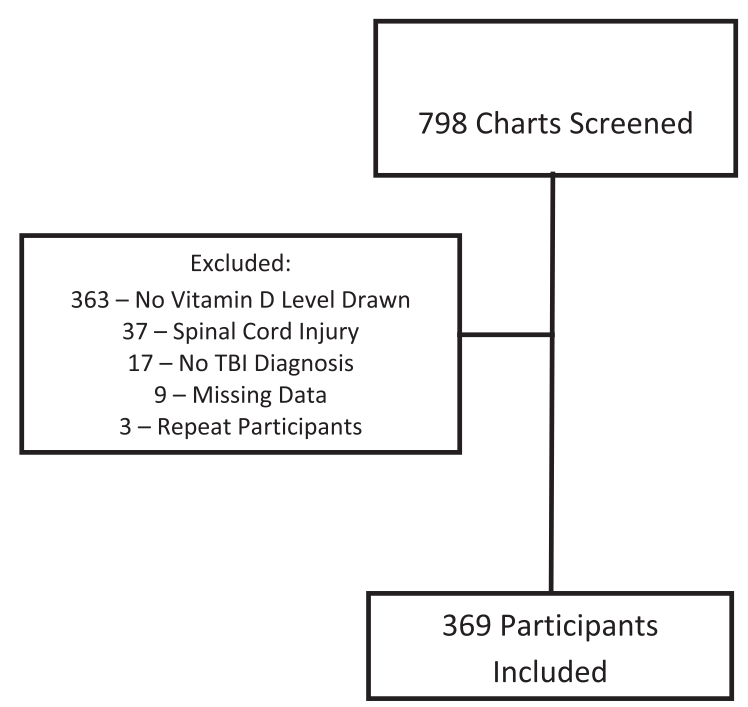

Fig. 1. Subject screening diagram.

patient's trauma and inpatient rehabilitation medical record and eRehabData ${ }^{\circledR}$.

\subsection{Measures/outcomes}

Demographic information was obtained for each patient, including race, ethnicity, age, gender, insurance coverage, and discharge disposition. Additionally, the following medical information was obtained from each patient's medical record: date of injury, mechanism of injury, length of stay in the rehabilitation hospital, Glasgow Coma Scale (GCS) score at initial hospital admission, FIM scores at admission and discharge to inpatient rehabilitation, and history of vitamin D deficiency and supplementation via history and physical documentation, medication and administration chart review.

\subsection{Statistical analysis}

Descriptive statistics were used to summarize demographic and injury characteristics. Patient data was then separated into three vitamin D groups according to vitamin $\mathrm{D}$ levels at admission to inpatient rehabilitation: deficient $(<20 \mathrm{ng} / \mathrm{mL})$, insufficient (20-29.9 $\mathrm{ng} / \mathrm{mL})$, or sufficient ( $\geq 30 \mathrm{ng} / \mathrm{mL}$ ). Comparisons of vitamin D groups and race were made using a Fisher's exact test. Comparisons of vitamin D groups and total FIM efficiency were made using analysis of variance. Spearman's correlations were employed to assess the relationship between
Table 1

Summary of demographic and clinical information

\begin{tabular}{|c|c|}
\hline & $\mathrm{N}=369$ \\
\hline Age, mean \pm sd & $43.5 \pm 21.0$ \\
\hline Male & $246(67 \%)$ \\
\hline Hispanic Ethnicity & $57(15 \%)$ \\
\hline \multicolumn{2}{|l|}{ Race } \\
\hline Asian & $4(1 \%)$ \\
\hline Native Hawaiian/Pacific Islander & $1(0.3 \%)$ \\
\hline Black or African American & $27(7 \%)$ \\
\hline White or Caucasian & $327(89 \%)$ \\
\hline Unknown/Missing & $10(3 \%)$ \\
\hline \multicolumn{2}{|l|}{ Primary Insurance Type } \\
\hline Private & $228(61 \%)$ \\
\hline Self-pay or uninsured & $3(1 \%)$ \\
\hline Medicaid & $7(2 \%)$ \\
\hline Medicare & $73(20 \%)$ \\
\hline Tricare & $7(2 \%)$ \\
\hline Other & $46(12 \%)$ \\
\hline Unknown/Missing & $5(1 \%)$ \\
\hline \multicolumn{2}{|l|}{ Discharge Disposition } \\
\hline Private Residence & $241(65 \%)$ \\
\hline Nursing Home & $37(10 \%)$ \\
\hline Adult Home & $3(1 \%)$ \\
\hline Hospital & $29(8 \%)$ \\
\hline Other & $58(16 \%)$ \\
\hline Unknown/Missing & $1(0.3 \%)$ \\
\hline Length of Stay, median (IQR) & $20(13-29)$ \\
\hline Days from Injury to Rehab & $19.5(11-36)$ \\
\hline \multicolumn{2}{|l|}{ Mechanism of Injury } \\
\hline Vehicular & $222(60 \%)$ \\
\hline Violence & $21(6 \%)$ \\
\hline Sports & $4(1 \%)$ \\
\hline Fall & $89(24 \%)$ \\
\hline Falling/Flying Object & $5(1 \%)$ \\
\hline Pedestrian & $16(4 \%)$ \\
\hline Other/Missing & $12(3 \%)$ \\
\hline \multicolumn{2}{|l|}{ GCS } \\
\hline Mild & $58(16 \%)$ \\
\hline Moderate & $26(7 \%)$ \\
\hline Severe & $136(37 \%)$ \\
\hline Unknown/Missing & $149(40 \%)$ \\
\hline Vitamin D Level & $29.5 \pm 16.1$ \\
\hline \multicolumn{2}{|l|}{ Vitamin D Category } \\
\hline Deficient $(<20)$ & $95(26 \%)$ \\
\hline Insufficient (20-29.9) & $131(36 \%)$ \\
\hline Sufficient $(\geq 30)$ & $142(39 \%)$ \\
\hline
\end{tabular}

vitamin $\mathrm{D}$ level and acute hospital and inpatient rehabilitation stays and patient characteristics.

\section{Results}

Demographic information for all 369 patients are summarized in Table 1 . The majority of patients were male (63\%), Caucasian (89\%), and used private insurance $(61 \%)$. Average age of participants was $43.5 \pm 21.0$ years old. The most prevalent mechanisms of injury were vehicular collisions (60\%), falls $(25 \%)$ and acts of violence (6\%). GCS at admission 
into the acute care hospital was missing from $40 \%$ of the inpatient rehabilitation admissions. Of the GCS scores recorded, the majority were in the severe range (37\%).

$25 \mathrm{OH}$ vitamin D levels were labeled as deficient $(<20 \mathrm{ng} / \mathrm{ml})$, insufficient $(20-29.9 \mathrm{ng} / \mathrm{ml})$, or sufficient $(\geq 30 \mathrm{ng} / \mathrm{ml})$ based on Clinical Practice Guidelines (Holick et al., 2011). The average vitamin D level at admission was $29.5 \pm 16.1 \mathrm{ng} / \mathrm{ml}[62 \%$ of patients were insufficient $(n=131)$ or deficient $(n=95)]$.

A significant difference in vitamin D level was found based on race/ethnicity ( $p=0.01$ ) (see Table 2). While $57 \%$ of non-Hispanic White patients were vitamin D insufficient of deficient, $71 \%$ of non-Hispanic Black and $79 \%$ of Hispanic patients were insufficient or deficient. Additionally, no statistical differences emerged between FIM efficiency scores and vitamin D level categories (Table 2).

365 out of 369 (99\%) patients did not have documented past medical history of vitamin D deficiency per the history and physical documentation and admission medication orders. 79 out of 95 (83.2\%) deficient patients and 94 out of 131 (71.8\%) insufficient patients were prescribed vitamin $\mathrm{D}$ treatment during inpatient rehabilitation stay. There was no correlation between vitamin $\mathrm{D}$ level and acute hospital $(r=-0.02 ; p=0.7733)$ or inpatient rehabilitation length of stay $(r=0.002 ; p=0.9692)$.

\section{Discussion}

The present study employed a retrospective chart review to investigate the prevalence of vitamin $\mathrm{D}$ insufficiency and deficiency in TBI patients within inpatient rehabilitation. Overall, results revealed 63\% of TBI patients were vitamin D insufficient or deficient, which is consistent with broader national prevalence estimates (Jamall et al., 2016). However, no estimates of vitamin D levels in an inpatient TBI rehabilitation population are available in the literature, thus this figure provides important information about prevalence rates in this setting.

Sufficient, insufficient, and deficient groups significantly differed in terms of race, with the largest proportion of Black and Hispanic patients presenting with insufficient or deficient levels. This result is consistent with demographic differences within the wider US population, where the highest rate of vitamin D deficiency is found in Black and Hispanic individuals (Forrest \& Stuhldreher, 2011). Differences in vitamin D deficiency rates between White, Black, and Hispanic groups may have implications in terms of screening, health care access, and use of supplementation or treatment. Health care providers should therefore be aware of at-risk groups within rehabilitation settings, who may benefit most from screening and treatment.

It was predicted that insufficient and deficient vitamin $\mathrm{D}$ levels would be associated with poorer functional outcomes, however, no correlations were found between FIM and vitamin D status. Due to the broad nature of FIM categories, it would be difficult to use this measure as the sole indicator of vitamin D's impact on overall health and functional improvement.

While the rate of vitamin D treatment was $76.5 \%$ for insufficient and deficient patients, it is worth noting that only 369 out of 798 (46.2\%) patients had their vitamin D level screened upon rehabilitation admission. This gap in screening may be due to variability of physician practices, partially attributable to a lack of evidence-based recommendations to screen TBI patients for vitamin D deficiency. The present study adds important prevalence rates to the evidence base.

\subsection{Limitations and future directions}

A few limitations exist for this study. First, this data represents only one institution, and vitamin D levels may vary across geographic regions (Kimlin, 2008).

Table 2

Comparison between race/ethnicity and FIM efficiency and levels of vitamin D

\begin{tabular}{lcccr}
\hline & Deficient & Insufficient & Sufficient & $p$-value \\
\hline Race/Ethnicity & & & & $\mathbf{0 . 0 1 4 5}$ \\
$\quad$ Non-Hispanic White & $64(23 \%)$ & $94(34 \%)$ & $116(42 \%)$ & \\
Non-Hispanic Black & $11(41 \%)$ & $8(30 \%)$ & $8(30 \%)$ & \\
Hispanic & $18(32 \%)$ & $27(47 \%)$ & $12(21 \%)$ & \\
Other & $2(18 \%)$ & $2(18 \%)$ & $7(64 \%)$ & \\
FIM Efficiency & & & \\
$\quad$ Motor & $1.7 \pm 1.1$ & $1.5 \pm 1.1$ & $1.6 \pm 1.2$ & 0.3976 \\
Cognition & $0.6 \pm 0.5$ & $0.5 \pm 0.4$ & $0.6 \pm 0.5$ & 0.2267 \\
Total & $2.3 \pm 1.4$ & $2 \pm 1.3$ & $2.2 \pm 1.7$ & 0.3942 \\
\hline
\end{tabular}


Additionally, data that was available for extraction was limited due to the retrospective nature, and other determinants such as premorbid vitamin D levels, injury severity (i.e., GCS), comorbidities and neuropsychological outcomes were not always available.

While evidence-based guidelines for screening and treating vitamin D in TBI patients in inpatient rehabilitation do not exist, our study finds the majority of TBI patients have deficient of insufficient vitamin D levels in the acute rehabilitation setting. Future efforts to establish an association of vitamin D and functional (motor and cognitive) status should be pursued among individuals with TBI both in the acute and post-acute setting in order to compel clinicians to routinely screen for and replace vitamin D.

\section{Conclusion}

Ultimately, results provide an estimate of vitamin D insufficiency and deficiency rates in TBI patients admitted to inpatient rehabilitation. These rates lend support to screening $25 \mathrm{OH}$ vitamin D level upon admission to inpatient rehabilitation settings. Screening may be especially important for those patients of an ethnic minority, as they are at increased risk of insufficient or deficient vitamin D levels. Finally, future research should continue to investigate prevalence of vitamin $\mathrm{D}$, relationships between vitamin $\mathrm{D}$ levels and functional measures, and outcomes following vitamin D replacement in the TBI population. In this way, researchers can continue to inform practice and provide the best care for patients undergoing inpatient rehabilitation.

\section{Acknowledgments}

This work was presented as a poster at the American Congress of Rehabilitation Medicine Conference, October 2016, Chicago, IL, USA.

\section{Conflict of interest}

The authors have no conflicts of interest to disclose.

\section{References}

Boonen, S., Bischoff-Ferrari, H., Cooper, C., Lips, P., Ljunggren, O., Meunier, P., \& Reginster, J.-Y. (2006). Addressing the musculoskeletal components of fracture risk with calcium and vitamin D: a review of the evidence. Calcified tissue international, 78(5), 257-270.
Buell, J. S., \& Dawson-Hughes, B. (2008). Vitamin D and neurocognitive dysfunction: preventing "D" ecline? Molecular aspects of medicine, 29(6), 415-422.

Centers for Disease Control and Prevention. (2014). Report to Congress on Traumatic Brain Injury in the United States: Epidemiology and Rehabilitation. Atlanta, GA.

Faul, M., Xu, L., Wald, M. M., \& Coronado, V. (2010). Traumatic brain injury in the United States. Atlanta, GA: national Center for injury Prevention and Control, Centers for disease Control and Prevention.

Forrest, K. Y., \& Stuhldreher, W. L. (2011). Prevalence and correlates of vitamin D deficiency in US adults. Nutr Res, 31(1), 48-54. doi:10.1016/j.nutres.2010.12.001

Gloth, F. M., \& Tobin, J. D. (1995). Vitamin D deficiency in older people. Journal of the American Geriatrics Society, 43(7), 822828.

Grammatiki, M., Rapti, E., Karras, S., Ajjan, R., \& Kotsa, K. (2017). Vitamin D and diabetes mellitus: Causal or casual association? Reviews in Endocrine and Metabolic Disorders, $1-15$.

Holick, M. F., Binkley, N. C., Bischoff-Ferrari, H. A., Gordon, C. M., Hanley, D. A., Heaney, R. P., \& Weaver, C. M. (2011). Evaluation, treatment, and prevention of vitamin D deficiency: an Endocrine Society clinical practice guideline. J Clin Endocrinol Metab, 96(7), 1911-1930. doi:10.1210/jc.20110385

Houston, D. K., Cesari, M., Ferrucci, L., Cherubini, A., Maggio, D., Bartali, B., \& Kritchevsky, S. B. (2007). Association between vitamin D status and physical performance: the InCHIANTI study. The Journals of Gerontology Series A: Biological Sciences and Medical Sciences, 62(4), 440-446.

Jamall, O. A., Feeney, C., Zaw-Linn, J., Malik, A., Niemi, M. E., Tenorio-Jimenez, C., \& Scott, G. (2016). Prevalence and Correlates of Vitamin D Deficiency in Adults after Traumatic Brain Injury. Clinical endocrinology, 85(4), 636-644.

Kiebzak, G. M., Moore, N. L., Margolis, S., Hollis, B., \& Kevorkian, C. G. (2007). Vitamin D status of patients admitted to a hospital rehabilitation unit: relationship to function and progress. American journal of physical medicine \& rehabilitation, 86(6), 435-445.

Kimlin, M. G. (2008). Geographic location and vitamin D synthesis. Molecular aspects of medicine, 29(6), 453-461.

Muscogiuri, G., Annweiler, C., Duval, G., Karras, S., Tirabassi, G., Salvio, G., \& Mascitelli, L. (2016). Vitamin D and Cardiovascular Disease: From atherosclerosis to myocardial infarction and stroke. International Journal of Cardiology, 230, 577-584.

Povlishock, J. T., \& Katz, D. I. (2005). Update of neuropathology and neurological recovery after traumatic brain injury. The Journal of head trauma rehabilitation, 20(1), 76-94.

Shan, N. L., Wahler, J., Lee, H. J., Bak, M. J., Gupta, S. D., Maehr, H., \& Suh, N. (2016). Vitamin D compounds inhibit cancer stem-like cells and induce differentiation in triple negative breast cancer. The Journal of Steroid Biochemistry and Molecular Biology.

Shinchuk, L. M., Morse, L., Huancahuari, N., Arum, S., Chen, T. C., \& Holick, M. F. (2006). Vitamin D deficiency and osteoporosis in rehabilitation inpatients. Archives of Physical Medicine and Rehabilitation, 87(7), 904-908.

Van der Schaft, J., Koek, H., Dijkstra, E., Verhaar, H., Van der Schouw, Y., \& Emmelot-Vonk, M. (2013). The association between vitamin D and cognition: a systematic review. Ageing research reviews, 12(4), 1013-1023. 